\title{
Measurements of electromagnetic fields in a planar radio-frequency inductively coupled plasma source
}

\author{
J. A. Meyer a) and A. E. Wendt \\ Engineering Research Center for Plasma-Aided Manufacturing, University of Wisconsin-Madison, \\ Madison, Wisconsin 53706
}

(Received 9 January 1995; accepted for publication 16 March 1995)

\begin{abstract}
Electromagnetic fields in a planar radio-frequency inductively coupled plasma source were measured using an inductive loop ( $B$-dot) probe. The probe was oriented to measure the time derivative of the axial component of the magnetic field $\left(\dot{B}_{z}\right)$. Using these measurements and Faraday's law, taking advantage of cylindrical symmetry, the time varying azimuthal electric field $\left(E_{\phi}\right)$ was calculated directly. Contour plots of $\dot{B}_{z}$ and $E_{\phi}$ in the $r-z$ plane show that the radio-frequency electromagnetic fields penetrate further into the plasma at lower gas pressure and lower rf power, corresponding to less effective shielding of the fields at lower plasma density. Estimates of skin depth from the axial decay of the field amplitudes near the axis of the discharge are consistent with values calculated from plasma parameters measured with Langmuir probes, confirming that near the axis the degree of shielding is most strongly dependent on the local plasma density. Near the conducting walls of the chamber, the skin depth calculations from the Langmuir probe data diverge from the $B$-dot probe data. $B$-dot probe measurements taken in the absence of plasma show that near the walls of the chamber the axial decay of the field amplitude is partly a geometrical effect in addition to a plasma shielding effect. (C) 1995 American Institute of Physics.
\end{abstract}

\section{INTRODUCTION}

Planar radio-frequency inductively coupled plasma (ICP) sources, also known as radio-frequency inductive (RFI) and transformer coupled plasma (TCP) sources, are currently of interest for many industrial applications such as etching and deposition of thin films. A recent review article by Hopwood ${ }^{1}$ outlines the state of the technology and applications. The importance of source characterization increases with increased use of ICP sources. Measurements of electromagnetic fields provide an important benchmark for simulations of ICP discharges, because of the difficulties of accurately accounting for the effects of all nearby conducting surfaces on the field. The measurements reported here were made in the same system under conditions identical to those for measurements of spatially resolved plasma parameters and optical emission reported elsewhere. ${ }^{2-4}$

Previous characterization studies of planar ICP discharges in argon include Langmuir probe measurements of plasma parameters, ${ }^{2,4,5}$ spatially resolved optical emission ${ }^{3}$ and electromagnetic fields. ${ }^{6}$ These studies and scveral modelling efforts ${ }^{7-10}$ show an azimuthal if electric field amplitude that is null on axis, increases radially to a maximum value under the antenna and then falls to zero at the radial boundary. Optical emission was found to peak in the region of peak electric field amplitude as energy coupling to the electrons is maximum there. The plasma density profile, however, seems to be governed by diffusion and peaks near the center of the discharge volume for the reactor geometry examincd in this study. ${ }^{2}$ The degree of shielding of the electromagnetic field can be expected to be sensitive to the local electron density and thus decrease with increasing radius. In addition, plasma density increases with pressure and power

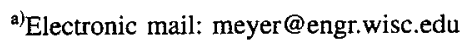

and, as a result, field penetration should decrease, with characteristic scale length given, for the conditions examined here, by the collisionless skin depth, $\delta$,

$$
\delta=\left(\frac{m_{e}}{\mu_{0} e^{2} n_{e}}\right)^{1 / 2}
$$

where $m_{e}$ is the mass of an electron, $\mu_{0}$ is the permeability of free space, $e$ is the charge of an electron, and $n_{e}$ is the electron density. ${ }^{11,12}$ It should be kept in mind the exponential decay associated with the definition of $\delta$ assumes a uniform concentration of charge carriers. Because of the strong gradient in $n_{e}$ at the edge of the plasma volume where the field is the strongest, this definition is somewhat ambiguous and we may expect some deviation from an exponential axial variation in the field amplitudes.

In the current work, the time derivative of the axial component of the magnetic field, $\dot{B}_{z}$, was measured versus axial and radial position over a range of discharge pressures and powers in argon. By using $\dot{B}_{z}$ rather than $\dot{B}_{r}$ as measured by Hopwood et al., ${ }^{6}$ the azimuthal electric field, $E_{\phi}$, may be calculated directly from Faraday's law without any approximations or knowledge of local plasma density, and as such should give a more accurate estimate of $E_{\phi}$.

\section{EXPERIMENTAL APPARATUS AND DIAGNOSTICS}

The planar ICP source used for these measurements is identical to that used in previous studies and has been described in detail elsewhere. ${ }^{2-4}$ Briefly, power from the four turn, $16.5 \mathrm{~cm}$ diameter, planar coil is coupled to the plasma through a radially spoked Faraday shield and a $1.27 \mathrm{~cm}$ quartz window. Gas is fed into the system through an annular gap around the edge of the quartz window. For our measurements, a liner was attached to the window flange to contain the plasma within a cylindrical volume. This grounded liner 
consisted of a $22.8 \mathrm{~cm}$ diameter by $13.7-\mathrm{cm}$-long metal cylinder with a circular end plate. The end plate had a $2.5 \mathrm{~cm}$ slot cut into it to facilitate probe measurements. The coil/gas feed/liner assembly was inserted into the end of a $35-\mathrm{cm}$ diameter by $61-\mathrm{cm}$-long vacuum chamber. The assembly was recessed into the chamber by a $12.5 \mathrm{~cm}$ vacuum well.

A magnetic induction $\left(B\right.$-dot) probe ${ }^{13}$ was used to measure $\dot{B}_{z}$ inside the cylindrical plasma volume. This probe consisted of a thin $50 \Omega$ coaxial cable with one end stripped to the inner conductor insulation. A loop in the inner conductor of $0.5 \mathrm{~cm}$ diameter was made and connected to the outer conductor to complete the circuit. This loop was bent $90^{\circ}$ so that the normal of the loop area was parallel to the cable. For all the measurements reported here the loop was oriented with its axis parallel to the axis of symmetry of the ICP system, so that only the axial component of the field contributed to the signal. The cable was inserted into a ceramic shaft which was attached to a mechanical probe driver. The loop was insulated from the plasma by a ceramic cap over the loop and the end of the ceramic shaft. The mechanical probe driver consisted of a rack and pinion assembly on the end of a linear vacuum feed through. This enabled the probe to be positioned to within $1 \mathrm{~mm}$ both axially and radially in the chamber, without breaking vacuum. Measurements were made every $1 \mathrm{~cm}$ axially between 0.5 and $6 \mathrm{~cm}$ and every 2 $\mathrm{cm}$ radially between 0 and $10 \mathrm{~cm}$. The signal was sent to an oscilloscope via a $50 \Omega$ terminated cable. All signal measurements reported here are peak-to-peak.

The voltage induced on the probe is given by $V=-d \Phi /$ $d t$, where $\Phi$ is the magnetic flux through the loop. For a single loop probe, $\dot{B}$ is then $-V / A$, where $A$ is the area of the probe. The magnitude of the magnetic field is given by $B=\dot{B} / \omega$, where $\omega$ is $2 \pi \times 13.56 \mathrm{MHz}=8.52 \times 10^{7} \mathrm{rad} / \mathrm{s}$. As an example, in Figs. 1 and 2, a $10000 \mathrm{~T} / \mathrm{s}$ contour line corresponds to $1.17 \times 10^{-4} \mathrm{~T}$. The electric field can be calculated by using the integral form of Faraday's law:

$$
\oint \mathbf{E} \cdot d \mathbf{I}=-\int \frac{d \mathbf{B}}{d t} \cdot d \mathbf{S}
$$

Using $\dot{B}_{z}$, and assuming cylindrical symmetry, the azimuthal electric field $\left(E_{\phi}\right)$ is then given by

$$
E_{\phi}(r)=\frac{1}{r} \int_{0}^{r} r^{\prime} \dot{B}_{z}\left(r^{\prime}\right) d r^{\prime},
$$

where $r$ and $r^{\prime}$ are the radial positions, as measured from the axis of the ICP system.

The calculation of $\dot{B}_{z}$ assumes that coupling to the probe is purely inductive. Because of the high voltages on the antenna, there was a concern about coupling of quasistatic electric fields interfering with the induction field, although these field are reduced by the presence of the Faraday shield. The effects of the capacitively coupled electric field on the probe were found by rotating the probe to measure $\dot{B}_{r}$ and positioning the probe on axis so that the magnetic field would be orthogonal to the probe area, but the capacitive electric field would still be strong. The signal on the probe was near zero, so we determined the capacitively coupled electric field did not affect the probe measurements.
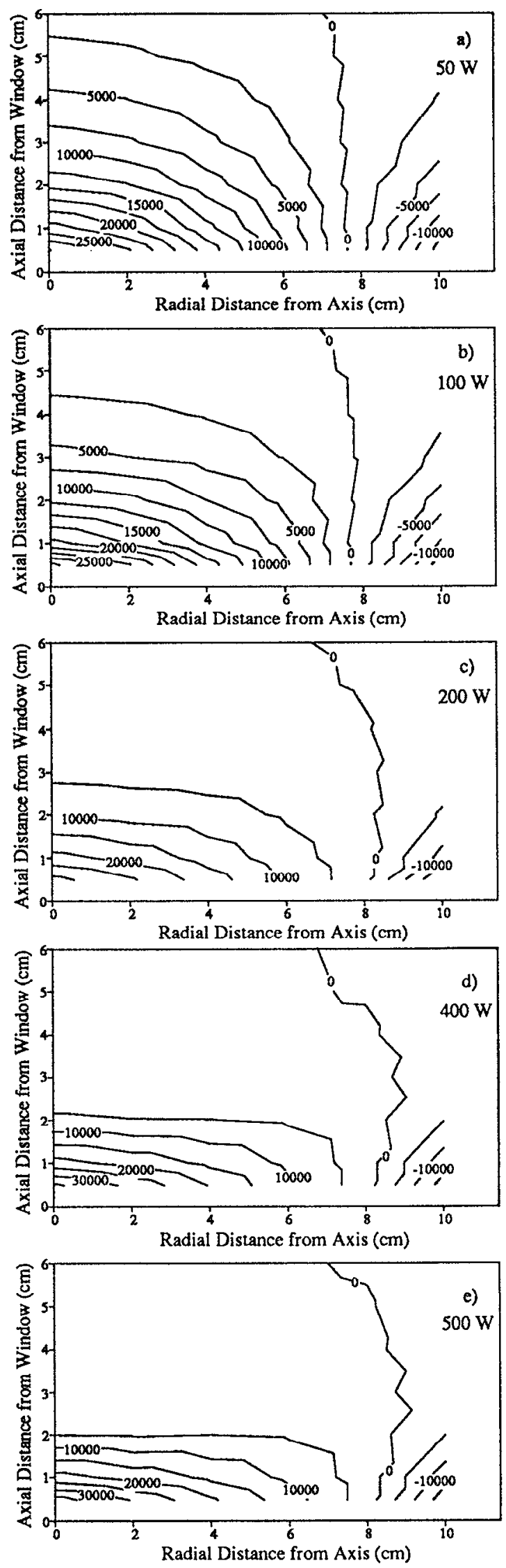

FIG. 1. The peak-to-peak magnitude of $\dot{B}_{z}(T / s)$ in a $10 \mathrm{mTorr}$ argon plasma for the following powers: (a) $50 \mathrm{~W}, 18 \mathrm{~A}$; (b) $100 \mathrm{~W}, 21 \mathrm{~A}$; (c) 200 W, $26 \mathrm{~A}$; (d) $400 \mathrm{~W}, 35 \mathrm{~A}$; (e) $500 \mathrm{~W}, 38 \mathrm{~A}$. 

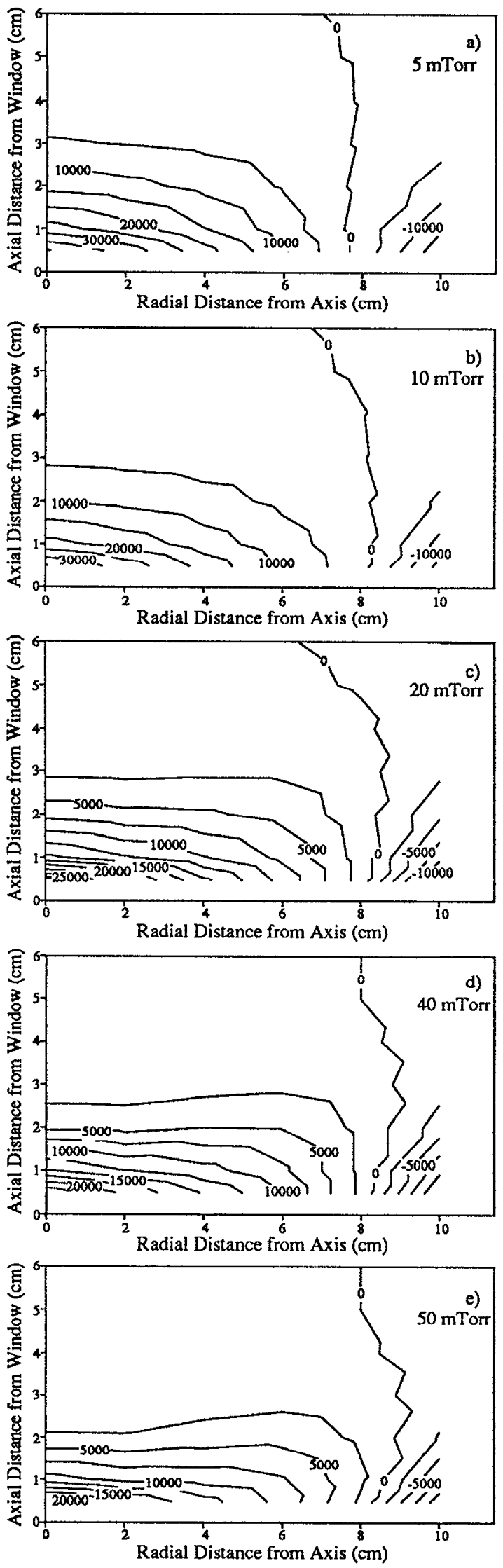

FIG. 2. The peak-to-peak magnitude of $\dot{B}_{z}(T / s)$ in a $200 \mathrm{~W}$ argon plasma for the following pressures: (a) $5 \mathrm{mTorr}, 28 \mathrm{~A}$; (b) $10 \mathrm{mTorr}, 26 \mathrm{~A}$; (c) 20 mTorr, $24 \mathrm{~A}$; (d) $40 \mathrm{mTorr}, 23 \mathrm{~A}$; (e) $50 \mathrm{mTorr}, 23 \mathrm{~A}$.

\section{RESULTS}

Figure 1 is a set of contour plots of $\dot{B}_{z}$ (peak-to-peak values) in the $r-z$ plane for several if powers in $10 \mathrm{mTorr}$ argon plasmas. Figure 2 is a set of contour plots of $\dot{B}_{z}$ in the $r-z$ plane for several operating pressures in $200 \mathrm{~W}$ argon plasmas. Negative numbers on the plots indicate a reversal in the direction of the field. The magnetic field intensity decreases as one moves further into the plasma from the antenna. At the higher powers and pressures, the contours tend to flatten radially and have higher axial gradients.

Contours of $E_{\phi}$ at different powers and pressures are shown in Figs. 3 and 4, respectively. The peak to peak values of $E_{\phi}$ are calculated from the $\dot{B}_{z}$ data in Figs. 1 and 2. We see, as expected, that the electric field amplitude rises from zero on axis to a maximum radially under the antenna and then falls toward zero at the radial boundary. However, the details of the spatial structure of the fields depend on the operating conditions. It can be seen that $E_{\phi}$ is markedly reduced at axial positions beyond $3 \mathrm{~cm}$ at higher powers and pressures as the density increases. In Fig. 4, it is apparent that the radial maximum in the electric field is moving outward as the pressure increases.

The distance an electromagnetic wave penetrates into a conductor is typically parameterized by the skin depth, the characteristic length scale for exponential decay. By estimating a skin depth for the ICP fields, we can quantify the degree to which they are being shielded by the plasma. However, there are two important differences between the situation examined here and the conditions under which the skin depth is defined. First, for the planar ICP system studied here, there is an axial variation of the fields even in the absence of a plasma, due to the geometry of the induction antenna. A second difference is that the derivation of the skin depth assumes wave propagation into a medium of uniform conductivity, where in this case the plasma conductivity (proportional to the electron density) varies dramatically in the relevant region. Some difficulty in inferring a skin depth from the data might be expected due to these deviations from ideal behavior. In fact, however, very good exponential fits to the data werc obtained, perhaps partly because the two effects described have opposite effects on the axial dependence and for these purposes may counteract one another.

The skin depth for ICP fields is found by fitting the axial dependence of $\dot{B}_{z}$ and $E_{\phi}$ to exponentials:

$\dot{B}_{z}(r, z)=\dot{B}_{z}(r, 0) e^{-(z / \delta)}, \quad E_{\phi}(r, z)=E_{\phi}(r, 0) e^{-(z / \delta)}$,

where $z$ is the axial position. At the positions where the skin depths were calculated for both $\dot{B}_{z}$ and $E_{\phi}$, an average was taken. Uncertainty occurs in the calculation of the skin depth when the data does not fit the exponential function well. Error bars were determined based on the residuals calculated from the fit.

The skin depth on axis $(r=0 \mathrm{~cm})$ versus $\mathrm{rf}$ power is shown in Fig. 5. The skin depth varies inversely with $\mathrm{rf}$ power. The skin depth on axis decreases with increasing operating pressure, as seen in Fig. 6. Figures 7 and 8 show the profiles of the skin depth across the radius of the device, and also includes values for fields measured in vacuum with $8 \mathrm{~A}$ 

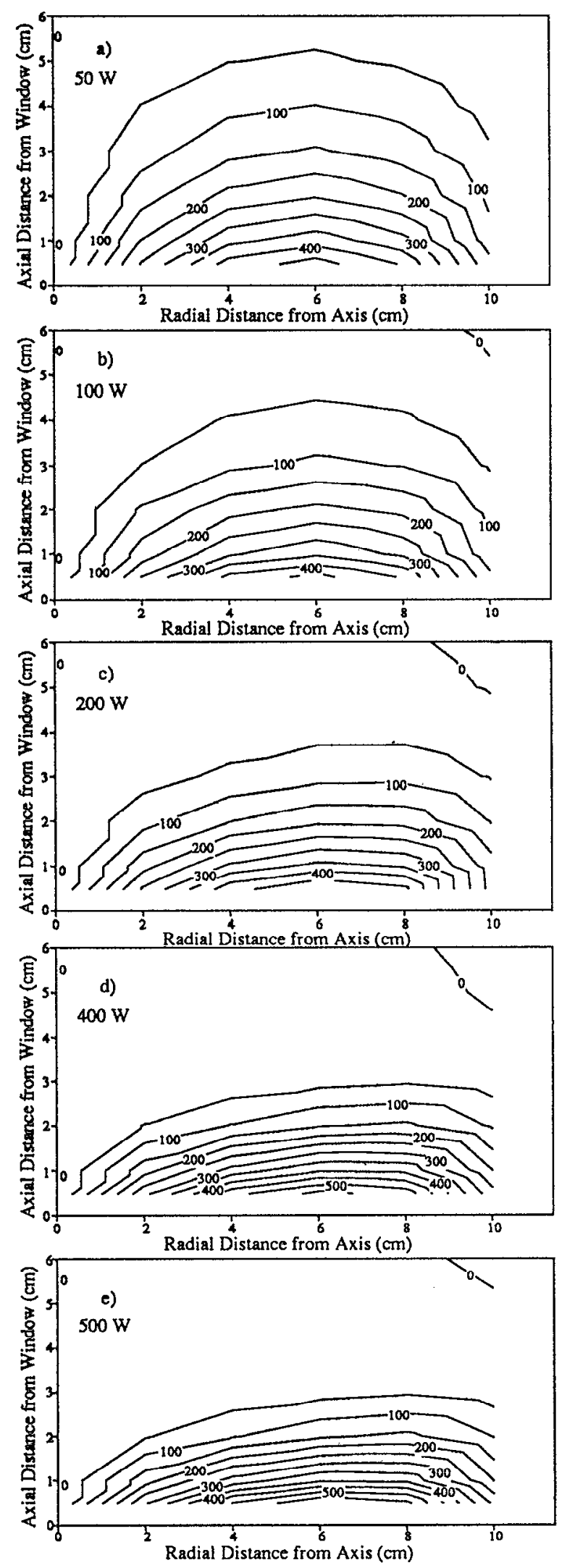

FIG. 3. The peak-to-peak magnitude of $E_{\phi}(\mathrm{V} / \mathrm{m})$ in a $10 \mathrm{mTorr}$ argon plasma for the following powers: (a) $50 \mathrm{~W}, 18 \mathrm{~A}$; (b) $100 \mathrm{~W}, 21 \mathrm{~A}$; (c) 200 W, $26 \mathrm{~A}$; (d) $400 \mathrm{~W}, 35 \mathrm{~A}$; (e) $500 \mathrm{~W}, 38 \mathrm{~A}$.
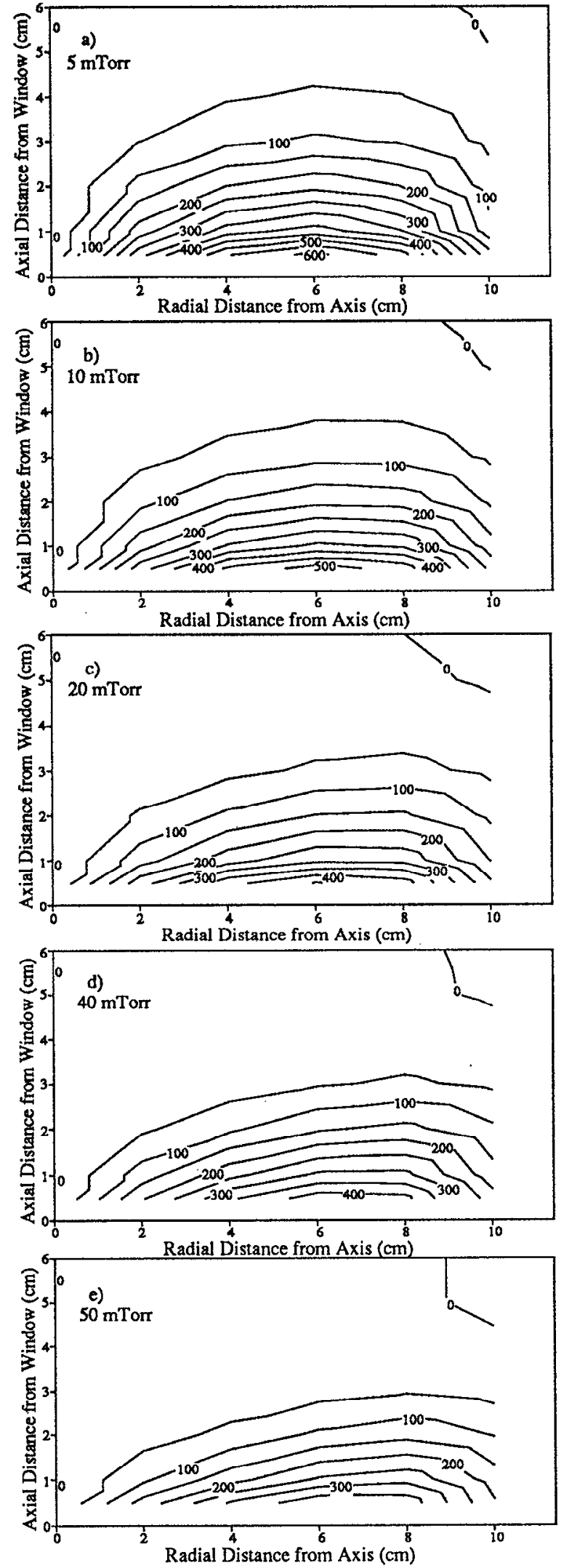

FIG. 4. The peak-to-peak magnitude of $E_{\phi}(\mathrm{V} / \mathrm{m})$ in a $200 \mathrm{~W}$ argon plasma for the following pressures: (a) 5 mTorr, $28 \mathrm{~A}$; (b) $10 \mathrm{mTorr}, 26 \mathrm{~A}$; (c) 20 mTorr, $24 \mathrm{~A}$; (d) 40 mTorr, $23 \mathrm{~A}$; (e) 50 mTorr, $23 \mathrm{~A}$. 


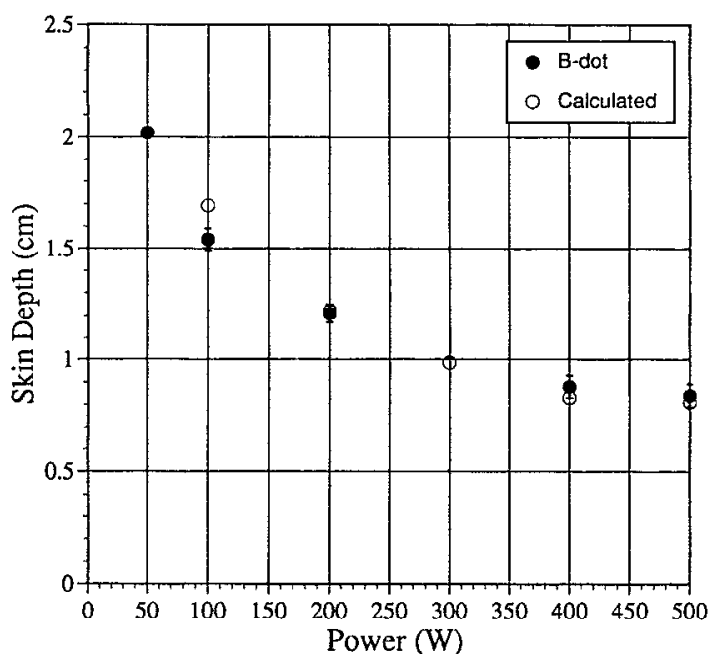

FIG. 5. The if skin depth on axis vs if power at 10 mTorr. The skin depths from $B$-dot probe data are compared to skin depths calculated from Langmuir probe density data taken at $z=4.4 \mathrm{~cm}$.

of rf current in the antenna. It should be noted the good exponential fits to the axial variation of the field amplitudes even in the absence of a plasma are due to geometrical effects. In addition, the fact that the skin depths determined in the vacuum case are always higher than with a plasma demonstrates the shielding effect of the plasma.

\section{DISCUSSION}

Peak plasma density (at the center of the discharge) increases both with increases in power and pressure. ${ }^{2,4,5}$ Two consequences of the shielding effect of the plasma on the electromagnetic field are illustrated in the contour plots. Both radial and axial changes in the spatial profiles of $\dot{B}_{z}$ and $E_{\phi}$ with pressure and power can be partially attributed to corre-

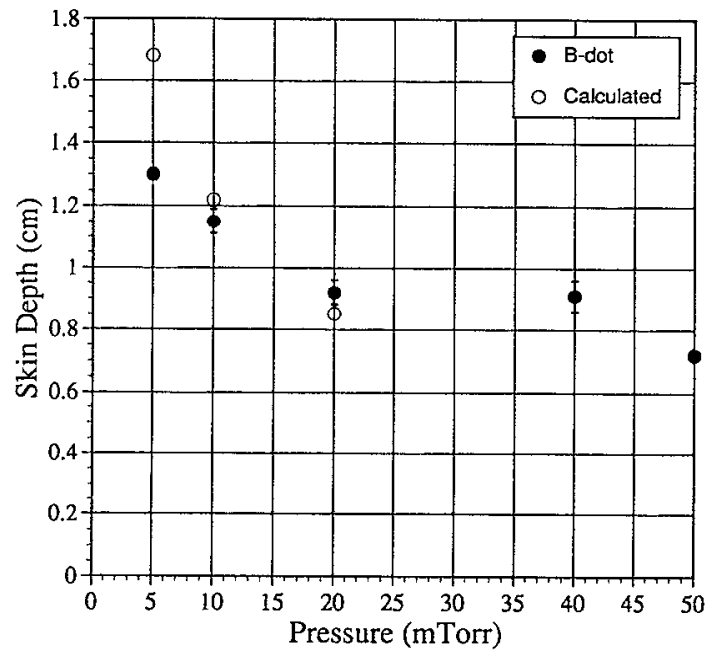

FIG. 6. The if skin depth on axis vs operating pressure at $200 \mathrm{~W}$. The skin depths from $B$-dot probe data are compared to skin depths calculated from Langmuir probe density data taken at $z=4.4 \mathrm{~cm}$.

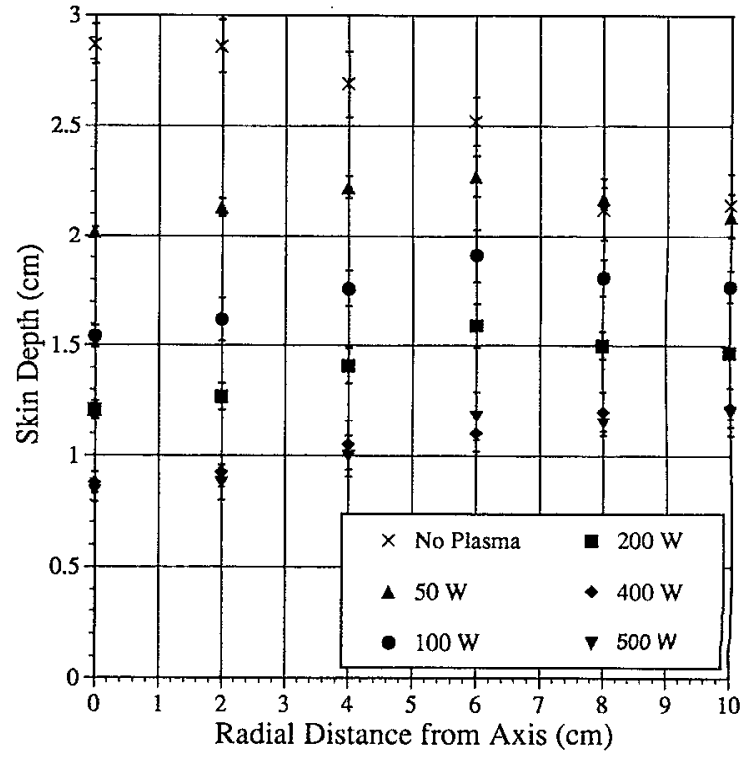

FIG. 7. The rf skin depth at several radii for the rf powers shown at 10 mTorr.

sponding changes in electron density, and therefore plasma conductivity. As electron density increases with both pressure and power, so does the plasma's ability to shield out the electromagnetic field. This results in an increased axial gradient in the field quantities with pressure and power, as seen in Figs. 5 and 6. However, because of radial variation in the plasma density as observed by Mahoney et al., ${ }^{2,4}$ the degree of shielding (axial field dependence) is also a function of the radial coordinate. Since the density is highest on axis, the shielding effect is greatest there. Furthermore, as the peak value of the density increases, so does the magnitude of the

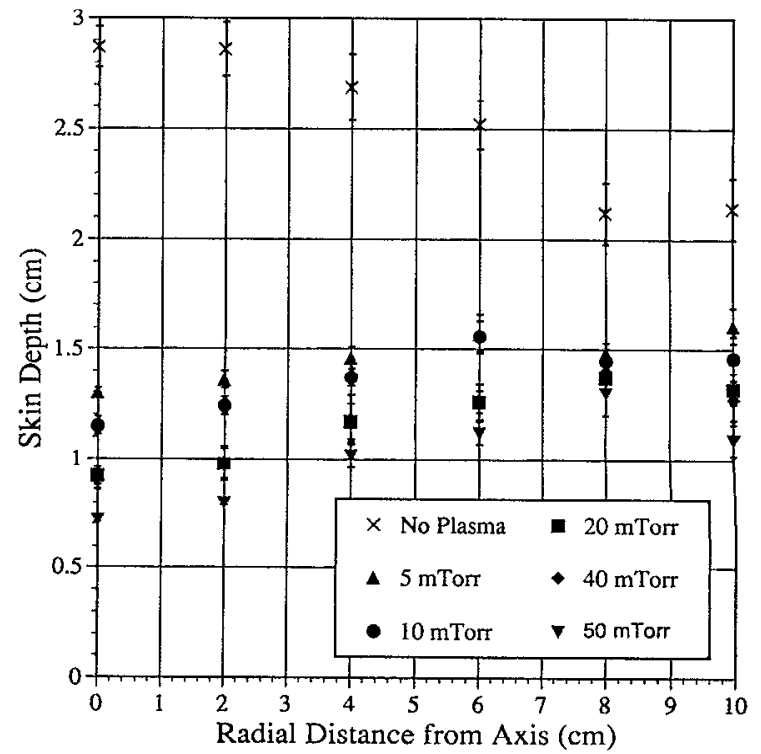

FIG. 8. The rf skin depth at several radii for the operating pressures shown at $200 \mathrm{~W}$. 


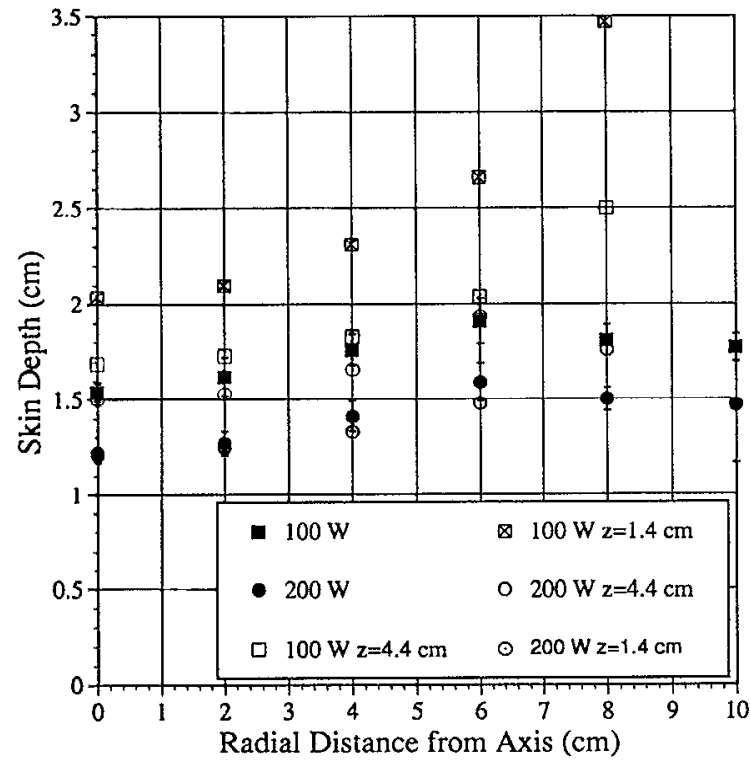

FIG. 9. The if skin depth at several radii for the 100 and $200 \mathrm{~W}$ of $\mathrm{rf}$ power at $10 \mathrm{mTorr}$. The skin depths from $B$-dot probe data are compared to skin depths calculated from Langmuir probe density data at two axial positions for each power.

radial density gradient $\left(\partial n_{e} / \partial r\right)$ and thus we sec increasingly more effective shielding on axis as compared to larger radii in Figs. 7 and 8.

From the data in Ref. 4, it becomes apparent that although increasing pressure and increasing power both have the effect of increasing the peak plasma density, the two parameters have different effects on the radial density profile. To be specific, the densities in the higher pressure cases tend to be more sharply peaked on axis, falling off steadily toward the radial boundary, while the higher power cases show a flatter profile near the center. For example, $10 \mathrm{mTorr}, 200 \mathrm{~W}$ and $20 \mathrm{mTorr}, 100 \mathrm{~W}$ cases from Ref. 4 have peak densities that are very close $\left(1.90 \times 10^{11} \mathrm{~cm}^{-3}\right.$ and $1.87 \times 10^{11} \mathrm{~cm}^{-3}$, respectively), but at a radius of $7.5 \mathrm{~cm}$, the difference in densities increased to $50 \%$, with the stronger radial gradient in this region being in the 20 mTorr case. This results in relatively strong variation in the degree of shielding of the fields with radius for higher pressures rather than higher powers, as also reflected in the rf field data in Figs. 5 and 6.

The radial dependence of the skin depths computed from the density data in Ref. 4 using Eq. (1) are compared in Figs. 9 and 10 to values obtained from the rf field data. Because of the strong axial density gradient in the region of strong fields, there is some ambiguity in the choice of axial location at which to evaluate the density for this calculation. Thus, skin depths were calculated for densities measured at both $z=1.4$ and $z=4.4 \mathrm{~cm}$. This axial range corresponds roughly to that over which the if field data were fit to an exponential, and gives a feel for the uncertainty. In all cases, the values of skin depth calculated from the density measured at $z=4.4$ $\mathrm{cm}$ are in good agreement with the if field data from $r=0$ out to $r=6 \mathrm{~cm}$. However, for radii larger than $r=6 \mathrm{~cm}$, the axial decay of the rf fields is much more rapid than predicted by the density data. This discrepancy can be explained by the

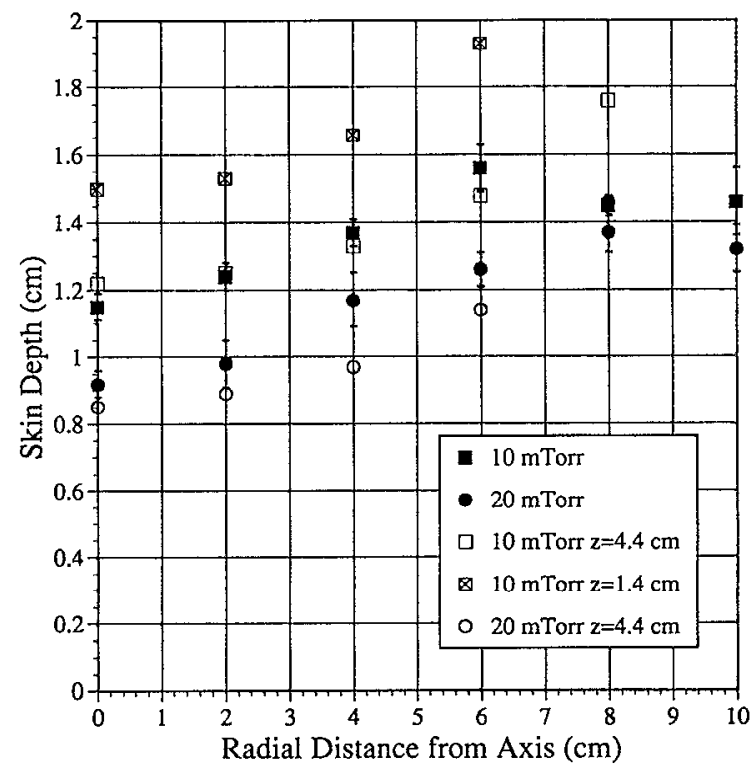

FIG. 10. The rf skin depth at several radii for operating pressures of 10 and 20 mTorr at $200 \mathrm{~W}$. The skin depths from $B$-dot probe data are compared to skin depths calculated from Langmuir probe density data at two axial positions at 10 mTorr and one axial position at 20 mTorr.

vacuum field data shown in Fig. 8. For larger radii, the skin depth calculated from the density data is close to or larger than that obtained from the vacuum field data, due solely to the geometry of the antenna and chamber. This suggests that at larger radii, the shielding effect of the plasma on the field is relatively weak, so that the axial decay of the field is dominated by the geometrical effect. However, because the skin depths obtained from the fields when the plasma is present are always lower than the vacuum values, it seems that shielding still makes some contribution to the axial dependence of the field amplitude.

\section{CONCLUSION}

Measurements of $\dot{B}_{z}$ were made using a $B$-dot probe in a planar ICP plasma source at several axial and radial positions in the device for several powers and pressures. From these data, $E_{\phi}$ at these positions, powers and pressures were calculated directly using Faraday's law. Both $\dot{B}_{z}$ and $E_{\phi}$ were analyzed and the rf skin depth was estimated using the axial decay of both field measurements. It was found that the rf shielding is reduced by both decreases in rf power and operating pressure, corresponding to decreases in the plasma density. Radial increases in the rf skin depth at radii close to the axis were found to correspond to radial gradients in the plasma density. Radial decreases in the rf skin depth at radii close to the wall were found to be due to geometrical effects.

\section{ACKNOWLEDGMENTS}

The authors would like to thank I eonard Mahoney and Robert Mau for their technical support. This work was supported by a grant from Lam Research Corporation and NSF Grant No. ECD-8721545 for the Engineering Research Center for Plasma-Aided Manufacturing. 
${ }^{1}$ J. Hopwood, Plasma Sources Sci. Technol. 1, 109 (1992).

${ }^{2}$ L. J. Mahoney, A. E. Wendt, E. Barrios, C. J. Richards, and J. L. Shohet, J. Appl. Phys. 76, 2041 (1994).

${ }^{3}$ D. F. Beale, A. E. Wendt, and L. J. Mahoney, J. Vac. Sci. Technol. A 12, 2775 (1994).

${ }^{4}$ L. J. Mahoney, Ph.D. thesis, University of Wisconsin-Madison, 1994.

5J. Hopwood, C. R. Guarnieri, S. J. Whitehair, and J. J. Cuomo, J. Vac. Sci. Technol. A 11, 152 (1993).

${ }^{6}$ J. Hopwood, C. R. Guarnieri, S. J. Whitehair, and J. J. Cuomo, J. Vac. Sci. Technol. A 11, 147 (1993).

${ }^{7}$ A. P. Paranjpe, J. Vac. Sci. Technol. A 12, 1221 (1994).
${ }^{8}$ G. DiPeso, V. Vahedi, D. W. Hewett, and T. D. Rognlien, J. Vac. Sci. Technol. A 12, 1387 (1994).

${ }^{9}$ P. L. G. Ventzek, T. J. Sommerer, R. J. Hoekstra, and M. J. Kushner, Appl. Phys. Lett. 63, 605 (1993).

${ }^{10}$ P. L. G. Ventzek, R. J. Hoekstra, and M. J. Kushner, J. Vac. Sci. Technol. B 12, 461 (1994).

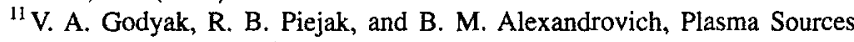
Sci. Technol. 3, 169 (1994).

${ }^{12}$ V. Vahedi, M. A. Lieberman, G. DiPeso, T. D. Rognlien, and D. W. Hewett (unpublished).

${ }^{13}$ R. H. Lovberg, in Plasma Diagnostic Techniques, edited by R. H. Huddlestone and S. L. Leonard (Academic, New York, 1965). 